reference group (OR: 5.1; 95\% CI 3.7 to 7.1). For the preterms the rate of TDS90 was higher for those with moderate/severe NDD (27/37 vs. 27/116, adjusted OR: 8.09 ; 95\% CI 3.2-19, and mild NDD 43/102 (adjusted OR: 2.2 (1.2-4.1). For preterms with no NDD TDS90 was more common than for the reference group (27/116 vs. $116 / 1089$, OR: 2.5 ; $95 \%$ CI 1.6 to 4.1 ).

Conclusion Extreme prematurity was associated with increased risk of later mental health problems, particularly if they had other neurodevelopmental disabilities.

\section{EVALUATION OF PREMATURE CHILDREN BY VOLUMETRIC MAGNETIC RESONANCE IMAGING AND COGNITIVE TESTS}

doi:10.1136/archdischild-2012-302724.1252

${ }^{1} E$ Arhan, ${ }^{2} \mathrm{~K}$ Gücüyener, ${ }^{1} \mathrm{ASS}$ Soysal, ${ }^{3} \mathrm{MA}$ Gürses, ${ }^{4} E$ Ergenekon, ${ }^{3} S ̧$ Şalvarll, ${ }^{4} \mathrm{C}$ Turkylmaz, ${ }^{4}$ E Koc, ${ }^{4}$ EE Onal, ${ }^{'} E$ Demir, ${ }^{1}$ A Serdaroglu, ${ }^{4} Y$ Atalay. ${ }^{1}$ Pediatric Neurology, Gazi University Faculty of Medicine; 'Pediatric Neurology, Gazi University Faculty of Medicine University; ${ }^{3}$ Radiology, INTEGRA Imaging Center; ${ }^{4}$ Neonatology, Gazi University Faculty of Medicine, Ankara, Turkey

Preterm children experience a high prevalence of long-term serious cognitive defects. Fetuses of 23 weeks of gestational age are now viable. Subsequent physiological stress can seriously disrupt the maturational processes that lay down this arthitecture. The ensuing abnormalities in brain may then contribute to the long term cogntive deficits. We aimed to measure regional brain volumes on the magnetic resonance imaging of prematurely born 9-year-old children and group matched term children. Nineteen nine year old preterm children and 21 term children recruited for the study. All subjectswent under the volumetric magnetic resonance imaging. In the neurocognitive assessment, it was observed that preterm children had impairments in visuospatial functioning, three- dimensional thought ability, data processing andlearning speed, executive function, complicated executive attention, perseveration, working memory, abstract thinking, installation replacement, focused attention. Volumes of serebellum, right and left caudate nucleus, right and left putamen, right and left globus pallidus. Right and left hypocampus and corpus callosum were significantly smaller in preterm children. There was a correlation between serebellar volume and executivefunction, harmony in social life, importance given to attentionand detail, visuospatial disorder and verbal IO, hypocampal volume and attention, arithmetics, verbal and performance IO, bilateral caudate nucleus volume and full scale IO score, vocabulary and speech skills, time in event processing, three dimensional thinking and verbal IO scores.

Our data indicate that preterm birth is associated with regionally specific, long term reductions in brain volume, in turn lead to poorer cognitive outcome.

\section{FA-VALUES IN THE PLIC AT TERM EQUIVALENT AGE ARE ASSOCIATED WITH NEURODEVELOPMENTAL OUTCOME AT TWO YEARS CORRECTED AGE}

doi:10.1136/archdischild-2012-302724.1253

K Kersbergen, N Wagenaar, B Van Kooij, F Groenendaal, LS de Vries, M Benders. Neonatology, Wilhelmina Children's Hospital/UMC, Utrecht, The Netherlands

Microstructural changes in white matter (WM) can be detected by Diffusion Tensor Imaging (DTI). This study tests the hypothesis that Fractional Anisotropy (FA) in the posterior limb of the internal capsule (PLIC), as measured directly on the FA-maps in preterm infants ATE is correlated to neuro-developmental outcome at two years corrected age.

Methods MRI at term was performed in 66 preterm infants (GA< 32 wks), Using colour-coded DTI maps, FA-values were assessed in the PLIC. Regions of interest (ROI's) were manually positioned in the PLIC bilaterally at the level of the foramen of Monro. FA-values from these ROI's were assessed in relation to scores for cognition, fine and gross motor outcome measured with the Bayley Scales of Infant and Toddler Development (BSITD)-III. Correlations were corrected for clinical factors.

Results FA-values in the left PLIC were correlated with cognition $(\mathrm{r}=0,435, \mathrm{p}<0.05)$, fine motor $(\mathrm{r}=0,335, \mathrm{p}<0.05)$ and gross motor $(r=0,337, p<0.05)$ outcome at two years corrected age. There was no such association for the right PLIC. After correction for gender, the correlation persisted only in boys. Postmenstrual age (PMA) and WMI were both significantly correlated with FA values in the left PLIC (resp $r=0.253, p<0.05$ and $r=-0.368, p<0.05$ ).

Conclusion FA-values measured by ROI-analysis ATE are significantly correlated with neurodevelopmental outcome at two years. This is in agreement with studies that used advanced postprocessing techniques to measure diffusion parameters. These measurements in this study are easy to perform, reproducible and directly applicable on MRI data and therefore particularly eligible for use in clinical care.

\section{LANGUAGE DEVELOPMENT OF PREMATURELY BORN CHILDREN - BIOLOGICAL DETERMINANTS AND SYNTAX}

doi:10.1136/archdischild-2012-302724.1254

'Z Kolundžić, ' ${ }^{2} \mathrm{M}$ Lenček, 'A Šimić Klarić. 'General Hospital, Požega; ${ }^{2}$ Faculty of Education and Rehabilitation Sciences, University of Zagreb, Zagreb, Croatia

There are numerous findings of the literature about premature born children having a higher degree of risk for different types of communication, language, reading and writing difficulties, compared to children born at term. These problems may seem minor in early language development and they are generally not recognized in that period. As the rule of language grows, particularly through reading and writing abilities, the problems become more evident and recognizable, and can significantly affect the academic achievement of children, especially if they do not provide help and support.

These theoretical findings were the basis for setting the aim of the study: to compare the outcome of prematurely borns in the age of 10 years, with the outcome of children born at term on linguistic tasks, especially in syntactic knowledge. According to this, one of the goals of the study was to investigate the relationship between biological variables (gestational age, birth weight, Apgar score) and linguistic- syntactic knowledge. The characteristics of this relationship were also examined. 34 prematurely born children and 34 children born at term participated.

Results showed statistically significant differences between prematurely born children and children born at term on syntactic knowledge tasks: prematurely borns' performings were significantly poorer. Biological factors were predictive for the level of syntactic knowledge. The purpose of this paper is to emphasize the importance of systematic monitoring of communication, language and speech development in prematurely born children.

\section{FUNCTIONAL ALTERATIONS OF THE HIPPOCAMPUS IN IUGR}

doi:10.1136/archdischild-2012-302724.1255

'M Camprubí Camprubí, 'JA Ortega Cano, 'C Duran Fernandez-Feijoo, 'I Iglesias Platas, ${ }^{3} \mathrm{~A}$ Ortega, ${ }^{1} \mathrm{X}$ Krauel, ${ }^{2} \mathrm{~S}$ Alcantara. ${ }^{1}$ Neonatology, Hospital Sant Joan de Déu, Universitat de Barcelona; '2Department of Experimental Pathology and Therapeutics, School of Medicine, University of Barcelona; ${ }^{3}$ Departamento de Celulas Troncales, CABIMER, Universidad Pablo de Olavide, Barcelona, Spain

Introduction Intrauterine growth restriction (IUGR) has been shown to relate to later neurodevelopmental problems. Recent studies suggest that deficits in spatial memory are the most prevalent among these individuals. The hippocampus, a key structure in spatial orientation, is susceptible to hypoxia or stress during pregnancy, 
as it happens in IUGR. To assess changes in neural connectivity in the hippocampus in IUGR animals, the hippocampus synaptic network has been analysed through three different synaptic proteins, Postsynaptic Density Protein 95 (PSD95), Synaptophysin and Synaptosome-associated Protein of $25 \mathrm{KDa}$ (SNAP25).

Methods IUGR was induced by meso-ovarian vessels' cauterization in pregnant rats. Sham surgery was performed in control animals. The pups were divided into: Control, Ischemic and IUGR (birth weight $<2$ SD). 25 days after birth, animals were subjected to an aquatic learning test. At day 35, they were sacrificed. Synaptic protein levels were analysed by immunochemistry staining and Western blotting.

Results There were differences in the learning outcomes between Control, Ischemic and IUGR animals. The analysis of PSD95, showed a gradual staining reduction from Controls to Ischemic to IUGR. There were no differences between groups in Synaptophysin inmunostaining. The intensity of SNAP25 staining was lower in Ischemic and IUGR than in Controls. These results were corroborated by western blot analysis.

Conclusions IUGR animals displayed reduced protein levels of PSD95 and SNAP25 in the hippocampus with respect to Control animals, suggesting a decrease in functional synapses.

\section{OUALITY OF GENERAL MOVEMENTS AFTER TREATMENT WITH LOW-DOSE DEXAMETHASONE IN PRETERM INFANTS AT RISK FOR BRONCHOPULMONARY DYSPLASIA}

doi:10.1136/archdischild-2012-302724.1256

MM Hitzert, ME van der Laan, AM Roescher, AF Bos. Division of Neonatology, Beatrix Children's Hospital, University Medical Center Groningen, Groningen, The Netherlands

Background Postnatal dexamethasone (DXM) is widely used to treat preterm infants at risk for bronchopulmonary dysplasia. Previously, it was reported that high-dose DXM leads to deteriorated quality of general movements (GMs). We determined neurological functioning in infants after low-dose DXM treatment, assessed by the GM-quality until three months post term.

Methods We included preterm infants, admitted to our NICU between 2010-2012 and treated with DXM (starting dose $0.25 \mathrm{mg}$ / $\mathrm{kg} / \mathrm{d}$ ). GM-quality was assessed before (day 0), during and after treatment until three months post term. We determined the change in GM-quality by comparing the GM-quality of day 0 with the GMquality of the last video recording. Additionally, we calculated a motor optimality score (MOS), ranging from 8 (low optimality) to 18 (high optimality).

Results Sixteen infants were included [median GA 26.9 wks (25.0-29.7); BW 800 g (620-1665)]. Before treatment, 4 infants had normal GMs which remained normal after starting treatment. GM-quality improved in 8 of 12 initially abnormal infants (Mc Nemar; $P=0.008$ ), whilst MOS slightly increased: median $10.5,12.0$ and 12.5 on days 0,1 and 7 , respectively (NS). Cumulative DXM doses, treatment duration and postnatal ages at starting DXM were not associated with change in GM-quality. Infants whose GMs improved were ventilated for a shorter period than infants whose GMs remained the same quality $(P=0.065)$.

Conclusions GM-quality did not deteriorate after DXM treatment but rather improved in infants with initial abnormal GMs. Our findings suggest that neurological functioning until three months post term is not adversely affected after low-dose DXM.

\section{THE NEURODEVELOPMENTAL ASSESSMENT OF VERY LOW BIRTH WEIGHT INFANTS AT 4-6 YEARS OF AGE}

doi:10.1136/archdischild-2012-302724.1257
'N Kavas, ${ }^{1} A E$ Arısoy, ${ }^{2} A$ Bayhan, ${ }^{3 B}$ Kara, ${ }^{1} A$ Günlemez, ${ }^{\prime} G$ Türker, ${ }^{1} M$ Oruç, ${ }^{1} A S$ Gökalp. ${ }^{1}$ Neonatology; ${ }^{2}$ Pediatrics; ${ }^{3}$ Pediatric Neurology, Kocaeli University Medical Faculty, Kocaeli, Turkey

The major and minor neurodevelopmental morbidities among premature infants become an important issue because of the increase in the number of surviving premature newborns.

The Aim of this study was to examine the cognitive, neuromotor, emotional and behavioral outcomes of the premature newborns at 4-6 years of age born with very low birth weight and to investigate the relationship between neuromotor and neurocognitive development.

The neuromotor status of 68 children were evaluated according to Touwen neurological examination, 64 children were assessed using Stanford-Binet and Peabody Picture Vocabulary Test, 65 children using Strength and Difficulties Questionnaire and Vineland Adaptive Behavior Scale.

Three cases were already diagnosed and followed as CP. According to Touwen examination 28 (\%41.2) children were normal, 35 (\%51.5) had simple minor neurological dysfunction (MND), 2 (\%2.9) had complex MND. The mean IQ score was 90.1 10.9 . The rate of hyperactivity, behavioral problems and emotional problems were in order $\% 60, \% 33.8$ and $\% 53.8$. The children were diagnosed as having a delay of $14.9 \pm 10.6$ month for conducting, 10.6 \pm 8.6 month for daily activities, $10.7 \pm 11.5$ month for social competence and a delay of $6.3 \pm 10.2$ month for motor behavior. The cognitive and neuropsychological results of the 35 children with MND and 28 children with normal neuromotor status were compared. The children with MND had significanly lower mean IQ score, more delay for motor behavior and they were more hyperactive.

Majority of the children who were considered as normal had cognitive impairment, language, behavior/emotional and neuromotor problems in various degrees.

\section{HEARING IN PRETERM INFANTS WITH POSTNATALLY ACOUIRED CYTOMEGALOVIRUS INFECTION}

doi:10.1136/archdischild-2012-302724.1258

IJ Nijman, ${ }^{2 B G A}$ van Zanten, 'AKM de Waard, 'C Koopman-Esseboom, 'LS de Vries, 'MA Verboon-Maciolek. 'Neonatology, University Medical Center Utrecht; ${ }^{2}$ Otorhinolaryngology and Head \& Neck Surgery, Rudolf Magnus Institute of Neuroscience, University Medical Center Utrecht, Utrecht, The Netherlands

Background and Aims Long-term sequelae of breast milkassociated cytomegalovirus (CMV) infection in preterm infants are insufficiently evaluated. We studied the hearing of preterm infants with postnatal CMV infection within the first and second year of life.

Methods Preterm infants (GA<32wks) admitted to our NICU between 2003 and 2011, and diagnosed with CMV infection using CMV PCR of urine at 40wks were included. Congenital infection was excluded in all. Hearing was tested using auditory brainstem response $(A B R)$ in the neonatal period and during the first and second year of life. Neurodevelopmental outcome was estimated using the Griffiths mental developmental scale (GMDS) at 18 months.

Results Eighty-eight preterm infants were diagnosed with postnatal CMV infection of whom four were lost to follow-up. All infants had normal hearing in the neonatal period. ABR-tests were performed in $64 / 84(76 \%)$ infants during the first year of life (median corrected age 7 months, range 2-11) and in 18/84 (21\%) infants during the second year (median corrected age 33 months, range 12-50). None of the infants developed SNHL. Mean GMDS score evaluated so far in 58/84 (69\%) infants at 15.8 months corrected age (range 13.0-21.0) was 104.4 (SD 9.9) and mean score of the language subscale was 16.7 months (SD 2.1). There were no differences in clinical data, cerebral ultrasonography results, viral load and GMDS scores between infants with hearing tests and non-tested infants. 